\title{
Le sigle nell'italiano contemporaneo come risultato di un processo di lessicalizzazione
}

\author{
Ettore Marchetti \\ Dipartimento di Francese e Italiano \\ Università del Texas, Austin \\ ettore@utexas.edu
}

\begin{abstract}
L'uso delle sigle: $u f o, F b i$, è sensibilmente aumentato negli ultimi anni. È prolifico in vari linguaggi settoriali (tecnica, informatica, politica), ha acquisito una discreta vitalità nel parlato e ancora di più nello scritto, in particolare nei testi giornalistici. In questo articolo affronto una ricognizione generale delle sigle (d'ora in avanti SI) dell'italiano contemporaneo dando conto dei meccanismi del passaggio da espressione sintagmatica a sigla (ad esempio l'eliminazione delle preposizioni: Federal Bureau of Investigation > Fbi), meccanismi che sono in verità non numerosi ed elementari. Tramite l'analisi particolare dell'impiego di alcune SI in testi giornalistici, intendo stabilire in che misura le SI si possano ascrivere alla categoria di elementi lessicalizzati. Ciò porta ad una inevitabile riflessione sulle potenzialità della lessicalizzazione, e sulla versatilità che rivela quando investe, come in questo caso, elementi che sfuggono alle classificazioni grammaticali canoniche. Il mio breve contributo mira a evidenziare la stabilità delle SI nel lessico italiano, i meccanismi d'integrazione e la rivisitazione del concetto di 'parola' che le SI suggeriscono. La disinvoltura con cui le SI vengono usate senza esplicitazione suggerisce la certezza da parte di chi scrive che il significato sia sempre chiaro; a questo riguardo penso che un'analisi sulla percezione del lettore e sulla sua capacità di usare le SI nel parlato sarebbe un interessante sviluppo dell'argomento.
\end{abstract}

Keywords: Sigle. Lessicalizzazione. Parola. Categoria grammaticale. Giornali online. 
Abstract: The use of acronyms: ufo, Fbi, has increased in the recent years. It is very prolific in some types of languages (i.e. politics, computer science). It is very common in spoken language and even more in some types of written texts, especially newspapers. This article gives account of the mechanisms that change a noun phrase into an acronym (i.e. Federal Bureau of Investigation $>$ Fbi), and then tries to establish whether acronyms can be considered as lexicalized items, that is as items that originally belong to a different grammatical category (syntax or morphology) and then become lexical. This also leads to a reflection on the potentiality of the lexicalization process, especially when it involves items that are not included in the canonical grammatical classifications. The present work aims at demonstrating the stability of the acronyms in the Italian lexicon, the level of their integration, and also the fact that they suggest to rethink the concept of lexical item. The tendency of newspapers to use acronyms more and more often suggests a high level of confidence in the readers' ability to understand and decode such particular lexical item. With this respect, a further analysis of how people perceive acronyms and whether they are able to use them in a proper way in daily life speech would be an interesting development of the topic.

Keywords: Acronyms. Lexicalization. Word. Grammatical category. Online newspapers.

Questo lavoro esplora in maniera generale le sigle dell'italiano contemporaneo, individuando i meccanismi generali del passaggio dall'espressione esplicitata (es: Giudice delle Indagini Preliminari) alla sigla (Gip); in secondo luogo descrive l'uso delle SI nei quotidiani online mettendo in risalto i meccanismi grazie ai quali si può parlare di lessicalizzazione. Quando si parla di SI, si parla di un aspetto dell'italiano sviluppatosi negli ultimi decenni, che suscita curiosità e interesse in primis per la frequenza d'uso in determinati 
linguaggi settoriali. La letteratura sull'argomento non è vastissima; in Klajn (1972) troviamo alcuni accenni nell'ambito di uno studio generale sugli influssi dell'inglese sull'italiano, e quindi l'autore tratta solo sigle inglesi, individuando nella conservazione della forma originale una delle caratteristiche fondamentali. Anche Giovanardi (2003) contestualizza il fenomeno nella sfera del contatto con l'inglese, con osservazioni sulla pronuncia, sulla grafia e su problemi legati alla trasparenza semantica. Registro poi una puntualizzazione di D'Achille (2003: 139) a mio avviso importante: le SI vanno considerate dei meccanismi che quindi, anche se non producono neologismi, implicano il coinvolgimento di un processo dinamico della lingua; tali meccanismi agiscono riducendo forme che fanno già parte del sistema lessicale. Siamo ancora nel campo della morfologia lessicale, ma non in quello formazione delle parole. Il contributo più articolato e più completo lo fornisce Lavinia Merlini Barbaresi (2007), che delinea le tipologia delle sigle nellitaliano contemporaneo, ne illustra la struttura e propone alcuni possibili sviluppi teorici quali una riflessione sulla produttività o sulla “...loro complessità (delle sigle e delle abbreviazioni in genere) come elementi lessicali-referenziali" (Merlini Barbaresi 2007: 46). Anche lei è d'accordo con d'Achille nello scartare lo status di neologismi per le sigle, ma ne evidenzia comunque l'importanza e la vitalità. L'articolo di Merlini Barbaresi è parte di un progetto più ampio sugli acronimi in inglese.

Dai contributi passati si evince quindi che, da una parte, l'argomento non è completamente trascurato, dall'altra che non ha avuto trattamenti monografici (fatta esclusione per Merlini Barbaresi). Gli interrogativi da porre sono secondo me i seguenti: è possibile individuare dei meccanismi generali in base ai quali le SI vengono usate? Possono essere viste come stadio finale di un processo lessicalizzazione? In base a queste considerazioni preliminari, gli scopi dell'articolo sono due: cominciare ad inquadrare il fenomeno delle SI 
cercando di darne conto anche dal punto di vista numerico, descrivere l'eventuale sistematicità del loro adattamento nelle strutture dell'italiano al fine di spiegarle in termini di cambiamento linguistico, e in particolare in termini di lessicalizzazione.

L'articolo è diviso in tre parti generali: ad un resoconto delle SI registrate nel lessico italiano e delle loro generali caratteristiche seguono i risultati dello spoglio dei quotidiani. Poi passo ad alcune considerazioni in termini di adattamento semantico, morfologico, e laddove è rilevante, anche fonetico. Infine verifico la compatibilità di tali risultati con alcune definizioni del processo di lessicalizzazione.

\section{Le sigle nell'italiano contemporaneo: ricognizione generale}

Sembra un'impresa titanica redigere un dizionario di SI, per la grande rapidità con cui alcune escono dall'uso, e per l'estrema facilità che impiegano altre ad entrarci. Cominciano a circolare on-line alcuni tentativi di classificazione. Ne cito uno, a puro scopo informativo: il Diæionario degli acronimi all'indirizzo www.glossari.it, parte di un progetto gestito dalla giornalista e traduttrice Maddalena Delli, che raccoglie circa 1500 voci; circa il $30 \%$ è costituito da SI inglesi, rispetto al $2 \%$ di SI francesi. Si tratta comunque di una raccolta largamente incompleta, mancando di molte voci di uso comune quali ad esempio $\mathrm{Tbc}, \mathrm{Tg}, \mathrm{ko}$.

Passando alle opere cartacee, nel 1999 si registra l'uscita del Diz̨ionario delle sigle di Rosetta Palazzi e Mary Gislon, edito da Sonzogno, che comprende anche tedesco e francese. Nello stesso anno, il Dizionario delle sigle e degli acronimi, a cura di Andrea Malossini, presenta un inventario di 8000 SI con 11000 significati diversi. Nel 2001 Enrico Righini redige il Dižionario di sigle, abbreviazioni e simboli, 10000 voci, edito da Zanichelli. In questa sezione farò riferimento a queste ultime due fonti. 
Per quanto riguarda il passaggio dalla struttura sintagmatica alla SI, il processo è molto semplice in casi che non hanno preposizioni, come ad esempio: Figc< <ederazione Italiana Gioco Calcio, Fiat $<$ Fabbrica Italiana Automobili Torino, Ogm< Organismo Geneticamente Modificato (prima lettera di ogni parola), Gestapo<GEheiem STAats POlizei, Gescal=GEstione CAse Lavoratori (prima sillaba). C’è poi il vasto insieme di SI che traducono un sintagma contenente almeno una preposizione, un articolo e/o una congiunzione. In questo caso, la trasformazione in SI avviene nella maggior parte dei casi tramite l'eliminazione di questi ultimi: Ici<Imposta Comunale sugli Immobili; Ior<Istituto per le Opere di Religione; Urss< Unione delle Repubbliche Socialiste Sovietiche. Per quanto riguarda le SI straniere, vengono importate senza modifiche, a conferma della grande disponibilità dell'italiano ad accogliere forestierismi: Aids, Nato, Usa. Nello scioglimento di queste SI si rivela la differenza sintattica tra le due lingue. Al contrario, in francese e in spagnolo si tende a riprodurre la sintassi nazionale anche nelle SI oltre che nelle parole straniere; invece che Aids, Nato e Usa si avranno così Sida, Otan (francese e spagnolo), EE.UU. (spagnolo).

Ci sono poi casi in cui la scelta delle iniziali è dettata da evidenti motivi di plausibilità fonologica e da un certo grado di arbitrarietà: Comecon $<$ Consiglio di Mutua Assistenza Economica (anche se non mancano le eccezioni: casi come Polstrada<Polizia stradale, Confcommercio, impiegano nessi consonantici anomali in italiano). La vasta gamma di meccanismi di riduzione include anche il passaggio attraverso la sostantivazione di aggettivi: Società Finanz̧iaria d'investimento $>$ Finanziaria d'investimento $>$ Fininvest, Società Finanzৃiaria meccanica $>$ Finanziaria meccanica $>$ Finmeccanica.

\subsection{Riepilogo}


Le SI sono riduzioni di lessemi complessi ${ }^{1}$. Per il meccanismo di riduzione, abbiamo l'eliminazione di preposizioni e la selezione della prima lettera di ogni parola o la prima sillaba. Le SI straniere sono in genere riportate nella forma originale. Nel passaggio dal sintagma alla SI non c'è slittamento semantico e il processo è istantaneo, non diacronico. Le SI rispettano i criteri della non-interrompibilità e della non-mobilità dei costituenti, e rispondono quindi alla definizione di 'parola' (Voghera 1994).

\section{Le sigle nei quotidiani on-line}

In questa sezione riporto $\mathrm{i}$ dati dello spoglio effettuato sui due quotidiani on-line più letti in Italia ${ }^{2}$ LaRepubblica e Il corriere della sera per un periodo di tempo che va dal 4 al 14 ottobre 2011. Ho preso in considerazione la prima pagina e le sezioni economia, salute e sport. Le SI impiegate sono 68, di cui 17 inglesi. La più frequente è $P d l<$ Popolo delle Libertà, con 10 occorrenze, seguita da Pm<Pubblico Ministero con 7 e Ue $<$ Unione Europea con 6. Per tutte le SI trovate e per il rispettivo sintagma rimando alla tabella 4 a p. 16.

Vediamo ora di entrare nel dettaglio dei meccanismi di adattamento dando conto della scelta dell'articolo, del genere, della categoria grammaticale e di questioni relative alla trasparenza e all'esplicitazione

\subsection{L'articolo}

Nelle SI che iniziano per vocale la tendenza generale è di seguire le regole vigenti per i sostantivi. Si registrano però alcune oscillazioni: Ue si trova sia con l': "Borse in recupero, attesa per l'Ue", sia con la: Trichet: " $L a U e$ agisca in fretta"; "Aut aut della Ue: abolite i minimi salariali". Nei casi in cui la SI è pronunciata lettera per lettera si può avere un articolo diverso da quello che si avrebbe se fosse sciolta. Questo accade in particolare in presenza di una

\footnotetext{
${ }^{1}$ Per la definizione di lessemi complessi seguo Voghera (1994:185): "La maggior parte delle trattazioni di semantica e lessicologia segnala l'esistenza di sequenze costituite da due o più parole che presentano una coesione interna semantica e/o sintattica tale che possono essere considerate un unico lessema,...".

${ }^{2}$ Dati Audiweb 2010
} 
-n- o di una -f- iniziale, che richiedono l'o la -d- eufonica in contesto fonosintattico: "L'Fbi avrebbe sventato un piano"; "Bc-Ue ed Fmi chiedono altri sacrifici ad Atene"; in caso di esplicitazione della SI sarebbe: il Federal Bureau of Investigation, il Fondo Monetario Internazionale. Per quanto riguarda il singolare e il plurale, le SI seguono il modello dei forestierismi, e quindi senza flessione, lasciando così all'articolo il compito di indicare il plurale. "Berlusconi riparla. Attacca $i$ pm". Per il genere, si segue quello del referente del sintagma; quando la SI è inglese, il genere scelto è il maschile: il suv, il dna, il laser.

Un tratto interessante è la frequenza d'uso dell'articolo, che presenta un certo grado di imprevedibilità. Ecco una tabella riassuntiva:

Tab. 1

\begin{tabular}{|c|c|c|c|c|}
\hline & Articolo & Esempi & $\begin{array}{l}\varnothing \\
\text { articolo }\end{array}$ & Esempi \\
\hline $\begin{array}{ll}\text { Uso } & \text { generale } \\
\text { dell'articolo } & \end{array}$ & 68 & $\begin{array}{l}\text { La Gdf } \\
\text { sequestra } \\
\text { la moto } \\
\text { cinese. } \\
\text { Berlusconi } \\
\text { riparla, } \\
\text { attacca i } \\
p m\end{array}$ & 54 & $\begin{array}{l}\text { Fiom: } \\
\text { ottobre } \\
\text { sciopero. } \\
\text { Iniziativa } \\
\text { contro } \\
\text { Unicredit }\end{array}$ \\
\hline $\begin{array}{lr}\text { Casi in cui viene } \\
\text { riportata } & \text { una } \\
\text { dichiarazione } & \text { di } \\
\text { associazioni, enti, } & \text { partiti }\end{array}$ & 6 & $\begin{array}{l}\text { Il Miur: } \\
\text { "Ecco i } \\
\text { nomi dei } \\
\text { colpevoli" }\end{array}$ & 8 & $\begin{array}{l}\text { Fmi: "Non } \\
\text { bastano } 109 \\
\text { miliardi" } \\
\text { Bce: }\end{array}$ \\
\hline
\end{tabular}




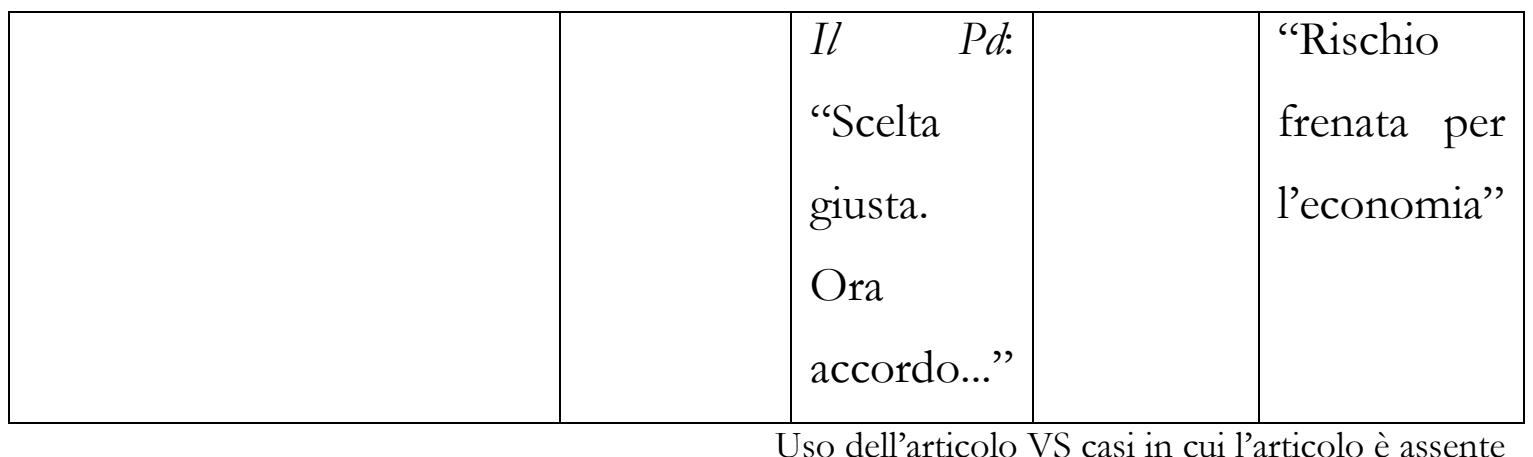

In contesti di discorso diretto, quindi, le SI sono considerate in 8 casi su 14 dei nomi propri, per sottolineare il fatto che un rappresentante dell'associazione/ente o partito sta parlando a nome di tutti, sfruttando il meccanismo della sineddoche. Gli altri esempi in cui si può non trovare l'articolo si spiegano plausibilmente con l'esigenza di concisione e economia del linguaggio giornalistico. L'unica certezza è data dai nomi comuni animati, per i quali l'articolo è usato nel 100\% delle occorrenze: "Il ct azzurro parla del match contro la Serbia", "L'ad Fulvio Conti rivede al ribasso la cedola", "Berlusconi riparla, attacca i $p m "$.

\subsection{Categoria grammaticale. Cambio di categoria grammaticale}

Le SI sono unanimemente assegnate alla categoria grammaticale del nome. Tale assegnazione avviene principalmente su base sintatticomorfologica. Nella seguente tabella sono riportati i dati riguardo ai settori rappresentati dalle SI e la distinzione in nomi propri e nomi comuni. Nella tabella 3 vediamo invece la distribuzione semantica:

Tab. 2

\begin{tabular}{|l|l|l|l|}
\hline $\begin{array}{l}\text { Totale } \\
\text { sigle }\end{array}$ & $\begin{array}{l}\text { Nomi } \\
\text { propri }\end{array}$ & $\begin{array}{l}\text { Nomi } \\
\text { comuni }\end{array}$ & Settori \\
\hline 68 & 49 & 19 & $\begin{array}{l}\text { Economia 5, salute 1, sport 2, } \\
\text { politica 11, }\end{array}$ \\
\hline
\end{tabular}




\begin{tabular}{|l|l|l|}
\hline & & $\begin{array}{l}\text { altro (ass. volontariato, finanza, } \\
\text { etc.) }\end{array}$ \\
\hline
\end{tabular}

Tab. 3

\begin{tabular}{|l|l|l|l|}
\hline Nomi propri & Esempio & Nomi comuni & Esempio \\
\hline Associazioni, & Wsj<Wall Street & Professioni, & Pm<Pubblico Ministero \\
organismi & Journal & oggetti & Pc $<$ Personal Computer \\
nazionali e & $\begin{array}{l}\text { Bce }<\text { Banca } \\
\text { internazionali, } \\
\text { compagnie } \\
\text { private, } \\
\text { banche }\end{array}$ & & \\
\hline Partiti politici & Pdl<Popolo della & & \\
& Libertà & & \\
\hline
\end{tabular}

Distribuzione semantica e funzioni grammaticali

La maggior parte delle SI rappresenta un nome proprio. Sono quindi altamente referenziali e mantengono la categoria grammaticale del lessema originario. Si verificano poi dei cambiamenti non definitivi di categoria. Il passaggio più comune è da nome ad aggettivo: "Il divo Usa", "Moderati Pdl verso lo strappo". Abbiamo poi l'inverso, cioè l'uso con la funzione di aggettivo sostantivato: "Frattini attacca gli ex $A n$ ", o in altri contesti non presenti nei testi da me esaminati: XL<extra large o $S<$ small, derivanti appunto da aggettivi, per indicare la taglia di un capo d'abbigliamento: "Questa maglietta è una XL". Si registra poi un caso di passaggio da nome inanimato a nome animato: Pr<Public Relations: "Mario fa il $p r$ in discoteca".

Un'ultima considerazione riguarda la flessione e la derivazione. Le SI che traducono nomi comuni non sono interessate dalla flessione né dalla 
derivazione, ma lo sono i rispettivi sintagmi di partenza, per lo meno dalla flessione: *pmi, *pmista, ma: Pubblico Ministero $>\mathbf{I}$ pubblic-i minister-i; ${ }^{*}$ cda-marca del plurale; *cda-suffisso derivativo, ma: Consiglio d'amministrazione> I consigli d'amministrazione. Le SI che traducono nomi propri possono dar vita a processi di derivazione, al contrario dei sintagmi di partenza: ciellino $<C l<$ Comunione e Liberazione; * *omunionino (per altri esempi di questo tipo si veda Thornton 2004).

\subsection{Trasparenza, esplicitazione}

Già Migliorini (1945) metteva in guardia sull'opacità di alcuni articoli di giornale per mancanza di spiegazione delle SI che venivano usate. Quello della trasparenza non è quindi un problema recentissimo, ma al momento attuale il dilagare di termini settoriali, tecnici, forestierismi, amplifica il senso di smarrimento. Probabilmente uno studio sulla percezione delle SI da parte dei lettori e sulla loro capacità di usarle nel modo appropriato rivelerebbe risultati interessanti.

A volte il contesto può non essere sufficiente a chiarire completamente il significato: "La Gdf sequestra la moto cinese"; "Gli assalitori sono curdi del Pkk". In altri casi, l'oscurità del senso della frase è totale: "Il Tas boccia il divieto imposto dal Cio". L'esigenza e le caratteristiche della comunicazione giornalistica portano ad utilizzare frasi di questo tipo che, sebbene di solito vengano chiarite nel corso dell'articolo ${ }^{3}$, scoraggiano la continuazione della lettura. Con molta probabilità le SI riconosciute senza esitazione sono quelle indicanti i partiti politici, le maggiori organizzazioni internazionali e le maggiori associazioni italiane. Secondo una personale sensazione senza nessuna conferma statistica, nella lista raccolta nei quotidiani circa il 50\%

\footnotetext{
${ }^{3}$ Un convincente elenco di meccanismi di esplicitazione delle SI nei quotidiani è elaborato da Merlini Barbaresi.
} 
sarebbero SI comprese dal lettore, senza bisogno di esplicitazione. Alcuni esempi: Spa, Inps, Iva, Irpef, G20, etc. Ci sono inoltre SI che mostrano tutte le caratteristiche fono-morfologiche delle parole e come parole sono percepite: gulag, yuppie, laser, ufo. Un ultimo dato: la media delle SI usate è di 19 occorrenze giornaliere in ciascun quotidiano (ricordo che non ho analizzato le edizioni complete, ma solo alcune sezioni).

Riepilogo: una prima osservazione riguarda l'uso dell'articolo non sempre prevedibile, e basato spesso su esigenze testuali (la sua omissioni nel riportare delle dichiarazioni); resta comunque il suo ruolo decisivo nell'indicare il numero o il genere del referente, visto che nelle SI non c'è flessione. L'impiego dell'articolo inquadra stabilmente le SI nella categoria del nome e nell'ambito del lessico, distinguendole in nomi propri e nomi comuni e confermandone un alto grado di autonomia. I sintagmi che originano le SI possono ammettere solo flessione, mentre le SI (quelle che indicano nomi propri) solo derivazione. Se equipariamo i sintagmi ai lessemi complessi, è possibile applicare queste osservazioni di Voghera: “...l’azzeramento della flessione facilita la cristallizzazione del sintagma fino alla sua lessicalizzazione..."; "Al contrario della flessione, la derivazione è un processo più lessicale...” (Voghera 1994: 205).

In assenza di contesto e di esplicitazione le SI sono semanticamente opache, diversamente da alcuni lessemi complessi: in esempi come ferro da stiro, se non conosciamo il significato dell'intera espressione ma sappiamo il significato dei singoli componenti possiamo risalire al senso generale. L'alto numero di SI nei giornali è segno di un certo grado di istituzionalizzazione.

\section{Definizioni di lessicalizzazione}

Le SI non costituiscono un fenomeno solo della lingua contemporanea (Migliorini 1949: 102), anche se il loro uso è cresciuto in maniera esponenziale 
negli ultimi anni; ma è corretto definirle un esempio di cambiamento linguistico e oggetto di lessicalizzazione? Tale azione può considerarsi affine a quella esercitata dalla lessicalizzazione sui lessemi complessi e sulle polirematiche (Voghera, Casadei)? L'eventuale lessicalizzazione nel caso delle SI, conferma la potenzialità di questo processo come fattore di cambiamento linguistico? Per cercare di rispondere, passo in rassegna alcune definizioni del processo di lessicalizzazione, e confronto la loro compatibilità con le considerazioni fatte precedentemente.

Quasi tutte le definizioni sono tratte da Brinton e Traugott 2005:

1) "Jacobson refers to a distinction between grammaticalization and lexicalization where both terms refer to strategies of linguistic coding (or, in a way, simply to grammar and lexicon) rather than to historical processes; in this sense, grammaticalized categories are said to be distinguished from lexicalized meanings by their obligatoriness" (Jacobson 1959a in Boretzky 1994, p. 534)

2) "La lessicalizzazione è uno dei possibili esiti dei processi di rianalisi funzionale di sequenze sintattiche da parte dei parlanti: essa permette l'entrata nel lessico di formazioni i cui componenti, perdendo la loro autonomia, sono percepiti come parti di un'unità lessicale" (Voghera 1994, pp. 188-189)

3) "In Moreno Cabrera's terminology, lexicalization is thus "syntactogenetic" (affecting syntactically determined words, phrases, or sentences) and lexicotelic" (going from syntax to lexicon) (Brinton and Traugott, p. 33)

4) "One of the most common conception of lexicalization is the unification or the univerbation of a syntactic phrase or construction into a single word" (Žirmunskij in Brinton e Traugott, p. 48) 
5) "... related to desyntacticization, in the sense of a syntagmatic structure losing its syntactic transparency and merging into a single lexical item" (Wischer in Brinton e Traugott, p. 48)

6) "A process by which complex words formally and semantically lose their motivation " or "a process by which complex words become simple words" (Blank in Brinton e Traugott, p. 48)

7) "The phenomenon that a complex lexeme once coined tends to become a single complex lexical unit, a simple lexeme. Through this process it loses the character of a syntagm to a greater or lesser degree" (Lipka in Brinton e Traugott, p. 48)

8) Brinton e Traugott rivisitano queste definizioni e propongono la seguente: "Lexicalization is the change whereby in certain linguistic contexts speakers use a syntactic construction or word formation as a new contentful form with formal and semantic properties that are not completely derivable or predictable from the constituents of the construction or the word formation pattern. Over time there may be further loss of internal constituency and the item may become more lexical" (p. 96).

Il punto che accomuna queste definizioni è il fatto che l'elemento lessicalizzato ha comunque un'origine sintattica. Inoltre il processo in generale non è reversibile, cioè un elemento che è riconosciuto come lessicalizzato non può riacquistare lo status precedente. In questo le SI sono differenti, in quanto possono coesistere con il sintagma che le origina. Ad esempio nei quotidiani, come si è visto, possono apparire nei titoli e poi nel corpo dell'articolo essere sostituite dal sintagma corrispondente.

Le descrizioni che comprendono tutte le caratteristiche delle SI sono la n. 1, la n. 3 , la n. 4 , la n. 5 e la n. 7 : 
-Jacobson ammette la possibilità di riferirsi alla lessicalizzazione non come ad un processo storico, ma come una strategia di codificazione: le SI sono un meccanismo di contrazione e non un cambiamento nel tempo. Moreno Cabrera evidenzia i due vertici del processo, nel quale il punto di arrivo è un elemento lessicale, in accordo con Žirmunskij che inoltre specifica: “...a single word". Il meccanismo di univerbazione che lo stesso Žirmunskij definisce è dal punto di vista formale perfettamente calzante con il fenomeno delle SI. Wischer si riferisce all'opacità dell'originario carattere sintattico, e abbiamo visto che spesso le SI vengono interpretate come vere e proprie parole, senza percepire il fatto che derivino da un sintagma (laser, ufo, yuppie). Infine, la definizione di Lipka è abbastanza generale da non porre particolari problemi.

Ad un livello di compatibilità parziale si attestano la n. 2 e la n. 6:

-Per quanto riguarda Voghera, l'unica caratteristica che non contraddistingue le SI sembra essere la rianalisi funzionale della sequenza di partenza, perché si è visto che non sono molti i casi di cambio della categoria funzionale da parte delle SI. Il fatto però che ci siano almeno alcuni casi di rianalisi non permette di escludere completamente l'applicabilità della definizione. Tutto sta nell'eventuale maggiore o minore futura produttività del meccanismo. Per il resto, le considerazioni di Voghera sul grado di lessicalizzazione raggiunto dai lessemi complessi (ferro da stiro, macchina da scrivere, fuggi fuggi, etc.) sono perfettamente applicabili alle SI, specialmente tenendo conto dei criteri di coesione morfosintattica da lei sfruttati. Voghera stabilisce quattro criteri generali di coesione morfosintattica in base al quale i lessemi complessi si distribuiscono: 1)lessemi con costituenti non mobili e non interrompibili 2)lessemi con costituenti non mobili ma interrompibili 3)lessemi con costituenti mobili ma non interrompibili 4)lessemi con costituenti mobili e 
interrompibili (197). Le SI, così come i sintagmi originari, appartengono al primo gruppo.

Blank infine pone il requisito della perdita di motivazione semantica, oltre che formale. Le SI non assumono un nuovo significato rispetto all'origine.

La n. 8, punto d'arrivo della riflessione di Brinton e Traugott nella loro monografia, enfatizza il cambio di significato come condizione necessaria della lessicalizzazione, che è comunque considerato un processo di cambiamento linguistico ed è diverso dai meccanismi di formazione delle parole. Questa descrizione oltre ad essere un compendio delle altre, le espande e le completa precisando la natura diacronica del fenomeno. Brinton e Traugott in generale escludono elementi come sigle e acronimi dall'ambito della lessicalizzazione, ma se si prende in considerazione la loro definizione sintetica, si può dire che le SI ne condividono alcune caratteristiche come ad esempio la progressione verso un maggiore peso lessicale (abbiamo visto: laser, yuppie) o la non prevedibilità dei singoli costituenti originari. Quest'ultima caratteristica raggiunge ovviamente il massimo grado nelle SI; consideriamo alcuni sintagmi originari: Pubblico Ministero o Disegno di Legge. I loro componenti presi singolarmente hanno un significato che subisce uno slittamento, più o meno profondo, se considerati in contesto sintagmatico. In altre parole, il significato totale dell'espressione non coincide con la somma dei significati dei singoli componenti, ma ha il tratto della non prevedibilità, così com'è definita da Brinton and Traugott. Al massimo livello di opacità si collocano le SI corrispondenti: $p m$, $d d l$. Questo esempio contribuisce a dimostrare la gradualità del processo di lessicalizzazione. 


\section{Conclusioni}

Il presente lavoro si proponeva uno sguardo d'insieme sulle sigle nell'italiano contemporaneo e quindi un esame di un caso in particolare, l'uso nei giornali on-line. Verificare se poi le SI siano in qualche modo interessate dalla lessicalizzazione deriva dall'intenzione di dar loro un inquadramento teorico. Le brevi considerazioni che seguono si basano su deduzioni empiriche e teoriche. Dai dati presi in esame emerge che le SI dellitaliano contemporaneo sono una riduzione di lessemi complessi, attraverso un passaggio istantaneo, non diacronico e che non subiscono cambi di significato. Il loro uso è in generale frequentissimo, quasi inflazionato nei quotidiani. Appartengono alla categoria grammaticale del nome e sono in maggioranza nomi propri. La loro stabilità nel livello del lessico, oltre ad essere sancita da motivazioni linguistiche quali l'uso dell'articolo, la possibilità della derivazione, o dall'alta frequenza d'uso, è corroborata anche da spinte sociolinguistiche come ad esempio la reazione del lettore, che le percepisce come parole, nel senso comune del termine, e le accetta in quanto tali. La possibilità per alcune di esse, secondo alcuni studiosi ben attestata, di procedere a derivazione, è un'ulteriore caratteristica lessicale, ed è inoltre uno degli aspetti su cui si attende con interesse di poter analizzare un maggior numero di dati. In base a questi elementi, le SI possono essere considerate e analizzate come stadio finale di lessicalizzazione.

Da un punto di vista teorico, si è visto come non ci sia una definizione univoca del termine lessicalizzazione nella sua completezza, ma c'è accordo solo sulle caratteristiche principali. Di conseguenza, trattandosi di un processo i cui confini non sono nettamente delimitabili, può comprendere un maggior numero di fenomeni, e nel caso in questione, le SI sono compatibili con buona parte delle definizioni considerate. Elementi come le SI, che hanno cioè 
uno status ibrido e una collocazione ancora incerta, sono dei nodi problematici tali da costituire degli ottimi esempi per verificare l'applicabilità di un approccio teorico, in questo caso la lessicalizzazione.

Lo studio delle SI mira a richiamare l'attenzione su un fenomeno molto vitale della lingua dei giornali, che ha ovviamente riscontro anche nel parlato quotidiano. Il tentativo di sistematizzare tale studio contribuirebbe a chiarire quali sono le potenzialità delle SI in ottica derivazionale, la produttività di alcune riduzioni occasionali (ad esempio il nome dei giornali stranieri: $W s j<W$ all Street Journal, Ft $<$ Financial Time), l'eventuale rischio di intaccare alcuni livelli della lingua come la fonologia. Da un punto di vista sociolinguistico, indagini sulla conoscenza delle SI e sull'uso che i parlanti ne fanno, aggiungerebbero una tessera importante agli studi sull'italiano dei nuovi media, una lingua che ha come caratteristica principale una serie di meccanismi di riduzione e di ellissi (e penso soprattutto agli sms, alle e-mail e alle chat) perfettamente in linea con le SI.

\section{Bibliografia}

ADAMO, G.; DELLA VALLE, V. (a cura di). Innovazione lessicale e terminologie specialistiche. Firenze: Olschki, 2003.

BORETZKY, N. et al. Dictionary of Grammaticalization. Bochum: Universitätsverlag Dr.N.Brockmeyer, 1994.

BRINTON J. L.; TRAUGOTT, E. C., Lexicalization and Language Change. Cambridge: Cambridge University Press, 2005.

CARDINALETTI, A.; FRASNEDI, F. (a cura di). Intorno all'italiano contemporaneo. Milano: FrancoAngeli, 2004. 
CASADEI, F. Le locuzioni preposizionali. Strutture lessicali e gradi di lessicalizzazione. Lingua e stile. Aprile, 43-80, 2001.

D’ACHILLE, P. L'italiano contemporaneo. Bologna: Il Mulino, 2003.

DARDANO, M.; TRIFONE, P. La nuova grammatica della lingua italiana. Bologna: Zanichelli, 1997.

GIOVANARDI, C.; GUALDO, R. Inglese-Italiano 1-1. Lecce: Manni, 2003.

KLAIN, I. Influssi inglesi nella lingua italiana. Firenze: Olschki, 1972.

LEHMANN, C. New reflections on grammaticalization and lexicalization. In New Reflections on Grammaticalization, a cura di WISHER, I. e DIEWALD, G. Amsterdam/Philadelphia: John Benjamins, 2002. pp. 1-18.

MALOSSINI, A. Dizionario delle sigle e degli acronimi. Milano: Vallardi, 1999.

MERLINI BARBARESI, L. Il fenomeno delle sigle: un primo schema di analisi. In Giuliana Garzone, Rita Salvi (a cura di). Linguistica, linguaggi specialistici, didattica delle lingue. Roma: CISU, 2007. pp. 37-47.

MIGLIORINI, B. Fisiologia e patologia delle sigle. In Accademia. 1, 31-35, 1945.

. Uso e abuso delle sigle. In Conversazioni sulla lingua italiana. Firenze: Le Monnier, 1956.

RIGHINI, E. Dizionario di sigle abbreviazioni e simboli. Bologna: Zanichelli, 2001. 
THORNTON, A. M. Riduzione. In GROSSMANN, M. e RAINER, F. (a cura di). La formazione delle parole in italiano. Tübingen: Niemeyer, 2004. pp. 555-566.

VOGHERA, M. Lessemi complessi: percorsi di lessicalizzazione a confronto. In Lingua e stile. 29 (2), 185-214, 1994. 